Caries Res. 1970;4:380

\title{
Subject Index Vol. 4
}

\section{Aluminium, 158}

Amylase, 193, 200

Apatite, 23

Blood, 347

Carbohydrate, dietary, 56, 114, 188, 332

Carbonate, dietary, 332

Caries, 49, 56, 78, 114, 124

diagnosis, 224

distribution, 69

fluoride-epidemiology, 293

inhibition, 269

rats, 49, 56, 63, 188, 332, 360 Diabetes, 78

Diet, 56, 114, 188, 332

Diffusion, 89

Enamel, 1, 158, 179, 206, 283

caries, 31

fluoride, 69

lesions, 224 Epidemiology, 78 Etching, 23

Experimental caries, 63, 131, 269 Fluoridation of domestic salt, 293 Fluoride, 1, 89, 131, 283

application, 63, 158, 269

analysis, 243

dentifrice, 149, 256

excretion, 256

in calculus, 168

enamel, 168

saliva, 321

silicate fillings, 168

- $\quad$ ingestion, 256

-, long-term effect, 149 -, mechanism of effect, 321

toothpaste, 149, 256

topical, 158

- $\quad$ uptake, 158, 168

Histopathology, 206, 360

Hungary, 293

Hydroxyapatite, 89

Levan synthesis, 97, 373

Microanalysis, 1

Mineralization, 31, 206 
Monkeys, 200

Morphology, 49

Neutron activation, 179 Nuclear magnetic resonance, 89 Nutritional requirements, 297, 305 Oral microbiology, 114, 297, 305 Phosphate, dietary, 332

hydrolysis, 14 Plaque, 97, 114, 243

biochemistry, 14

polysaccharides, 97, 114, 360

Radiographs, 224 Rat, 347

- $\quad$ caries, vide Caries, rats

Renal calculi, 332

Reversibility, 131

Saliva, 193

Salivary glands, 200 Scanning electron microscopy, 23 Selenium, 179 Starch, 193

Streptococci, 97, 114, 124 Streptococcus mutatis, 114, 124 Streptococcus salivarius, VIA, 360

Streptococcus sanguis, 114, 297 Synthetic media, 297, 305

Tooth development, 49, 56 Topical fluoridation, 63, 158 Transmissibility, 124 